\title{
Supporting Information: Energy Landscapes for Electronic Structure
}

Hugh G. A. Burton ${ }^{1,2, \text { a) }}$ and David J. Wales, b)

1) Physical and Theoretical Chemical Laboratory, University of Oxford, South Parks Road, Oxford OX1 3QZ, U.K.

${ }^{2)}$ Department of Chemistry, University of Cambridge, Lensfield Road, Cambridge, CB2 1EW, U.K.

(Dated: 24 November 2020)

\section{SI. UHF ORBITAL REARRANGEMENTS}

A. $m_{s}=1$

(i)
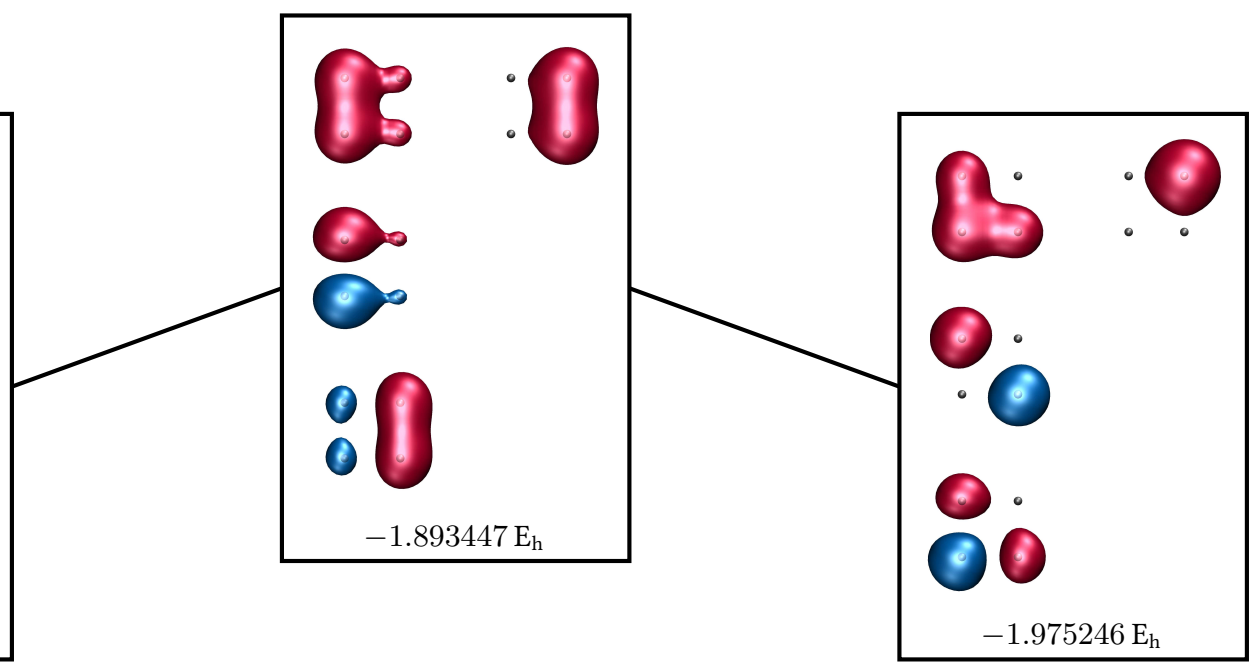

(ii)
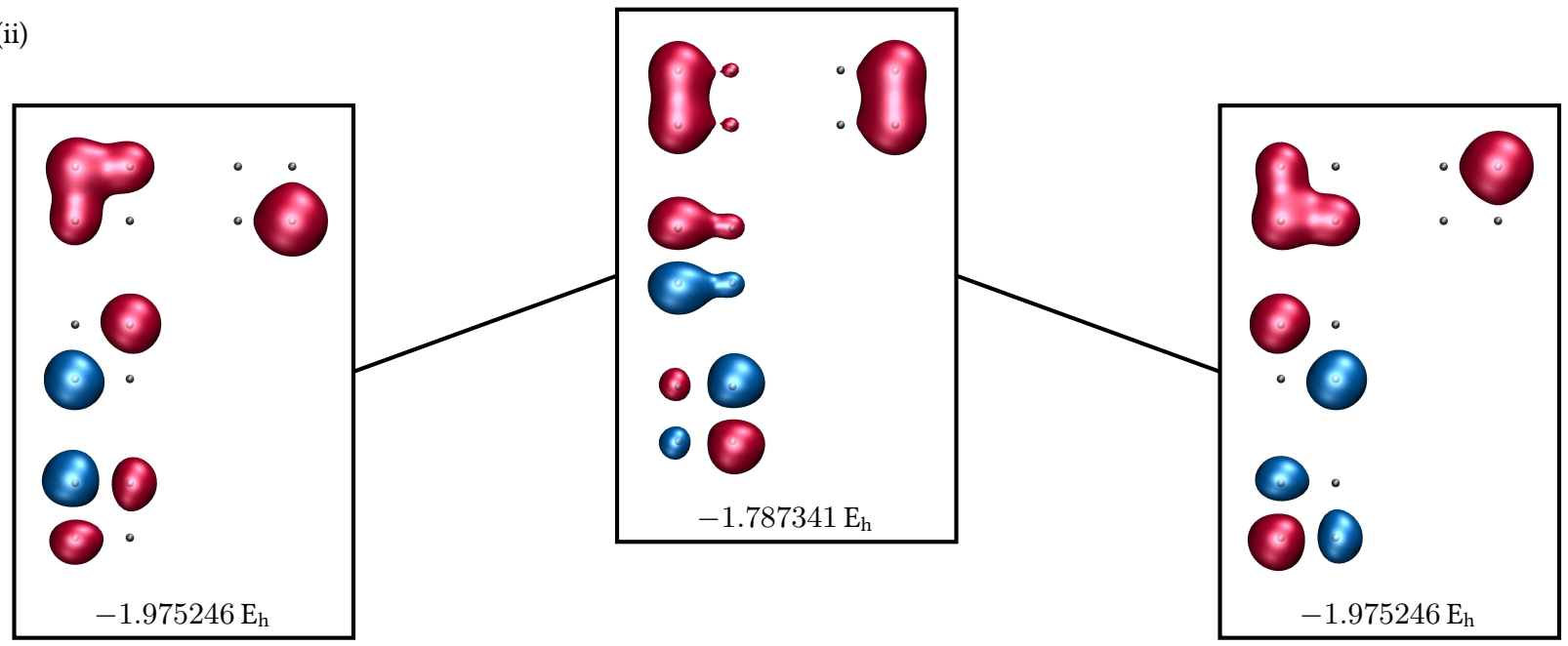

FIG. S1: Illustration of the orbital rearrangements for the UHF solutions of square $\mathrm{H}_{4}$ with a side length of $2.0 \AA$ and $m_{s}=1$. High-spin $(\alpha)$ and low-spin $(\beta)$ orbitals occupy the left and right columns respectively.

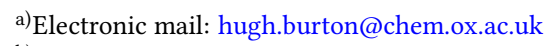

b)Electronic mail: dw34@cam.ac.uk 
(iii)

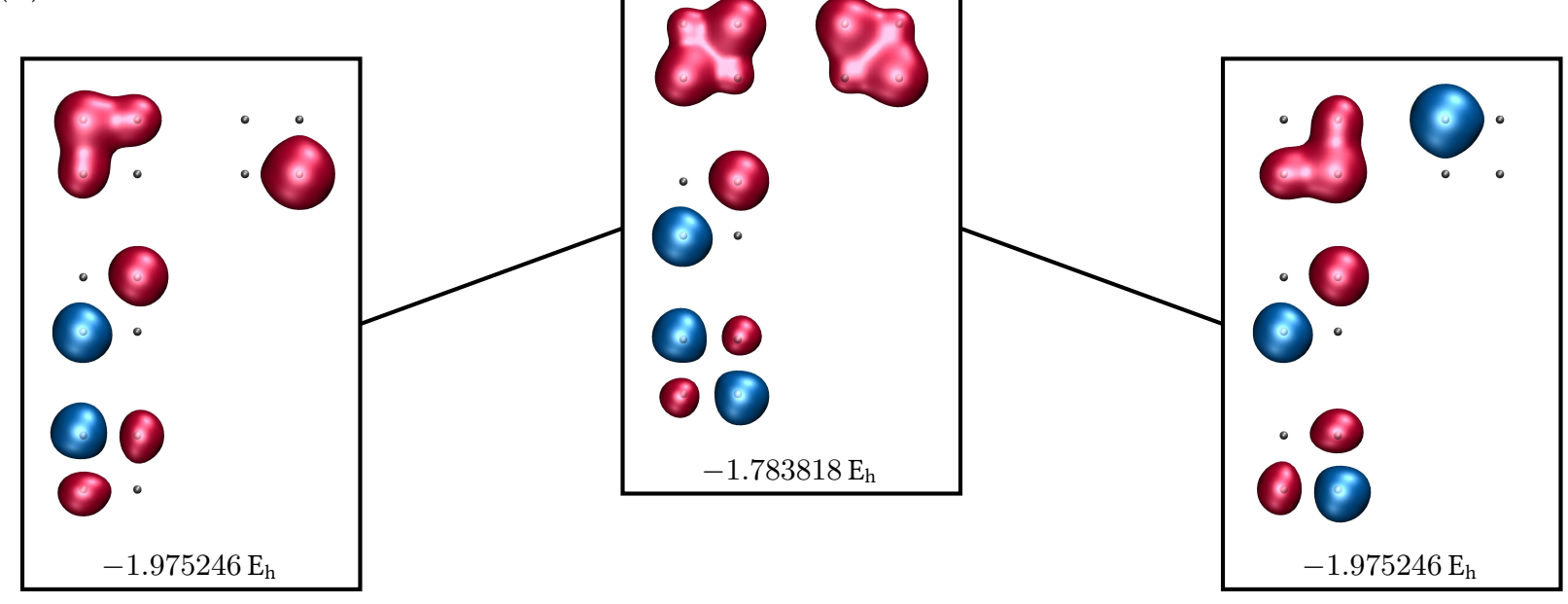

(iv)
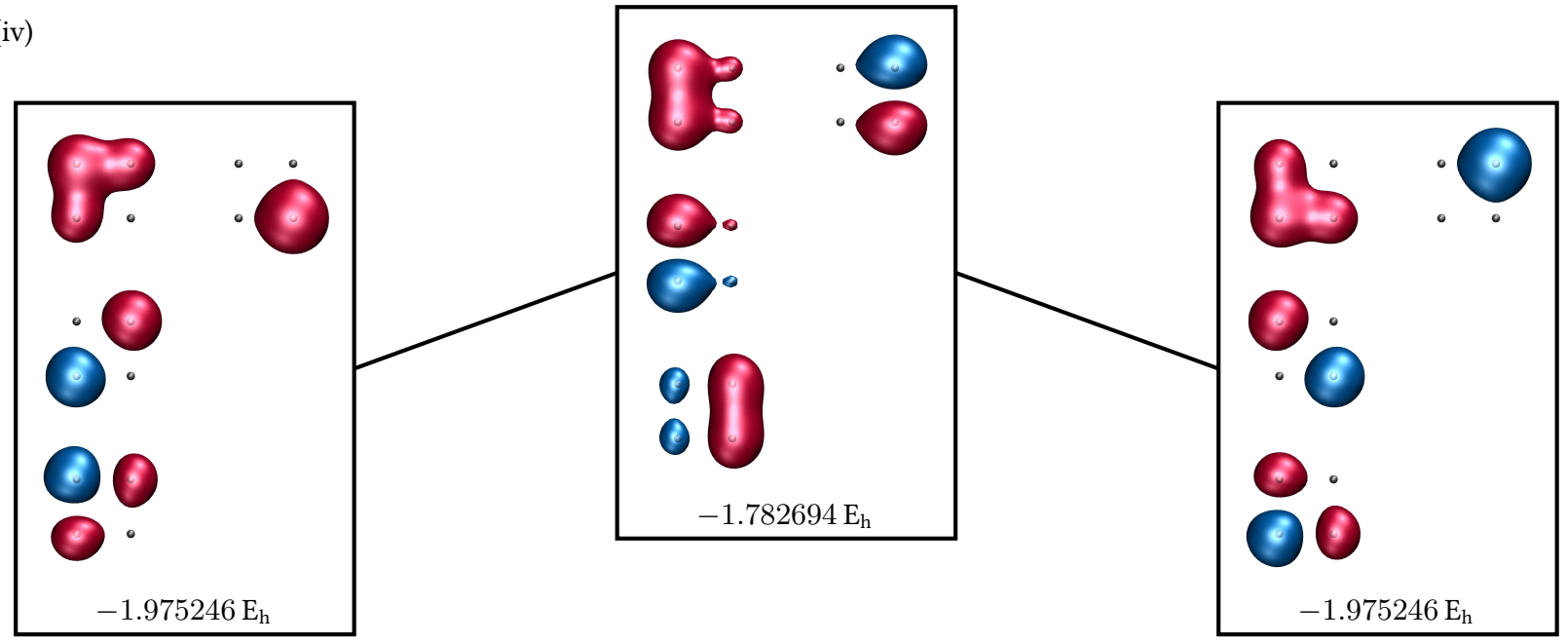

$(\mathrm{v})$
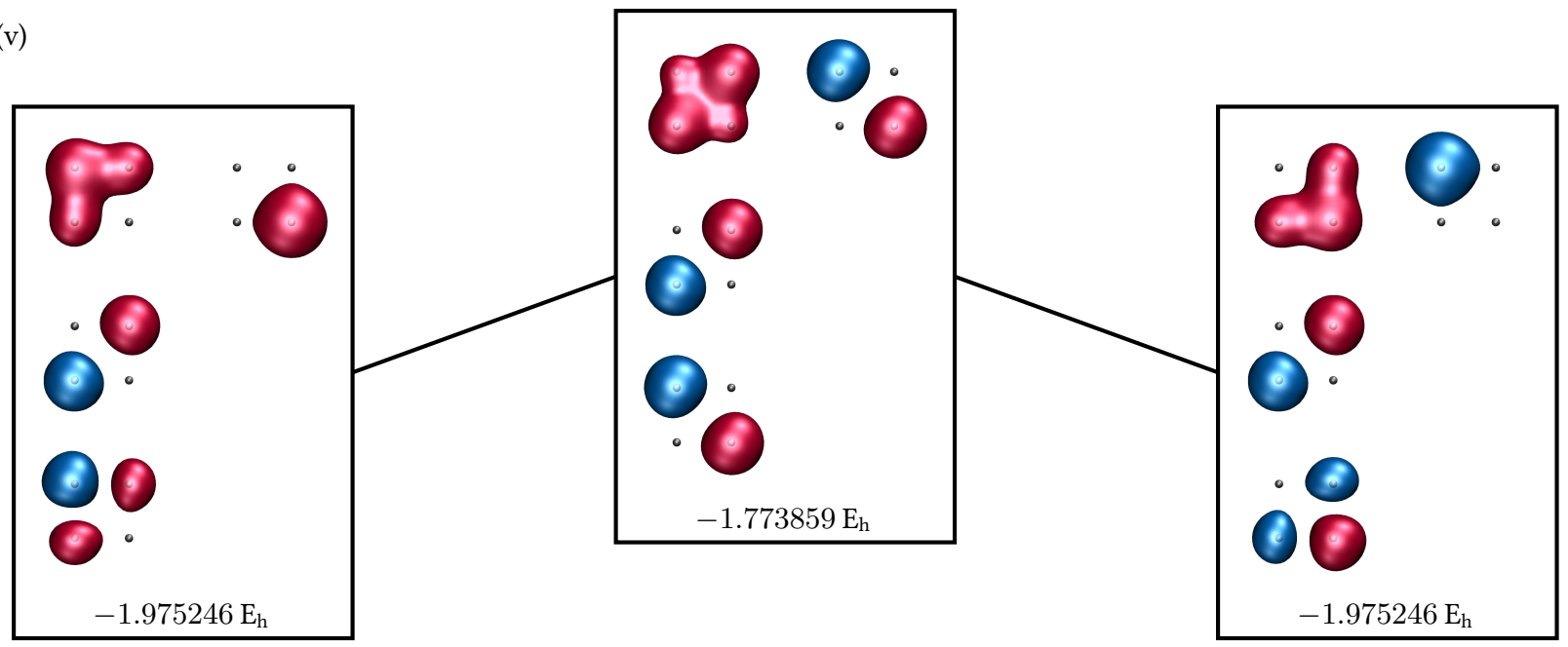

FIG. S1: (cont.) Illustration of the orbital rearrangements for the UHF solutions of square $\mathrm{H}_{4}$ with a side length of $2.0 \AA$ and $m_{s}=1$. High-spin $(\alpha)$ and low-spin $(\beta)$ orbitals occupy the left and right columns respectively. 
(vi)
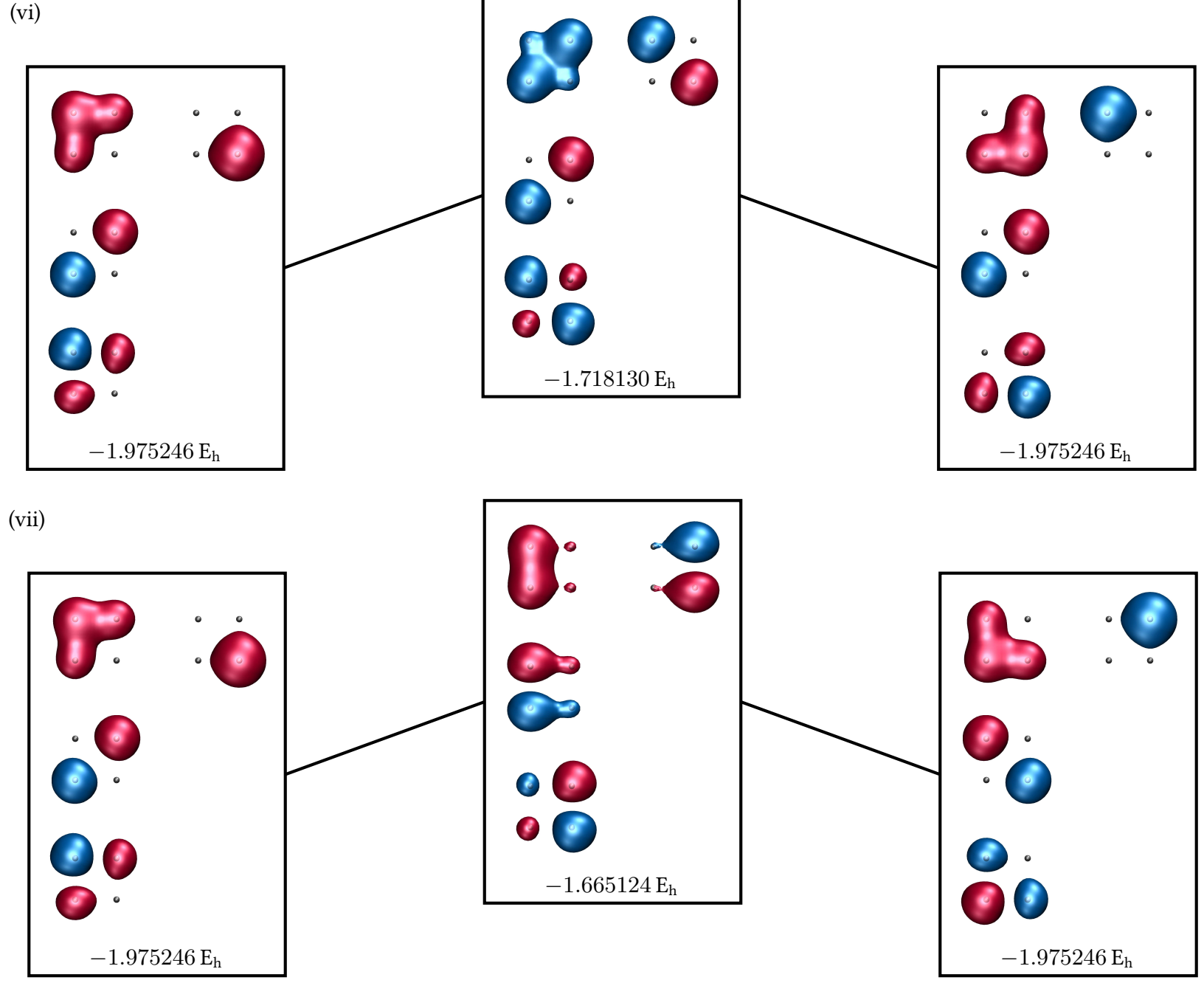

FIG. S1: (cont.) Illustration of the orbital rearrangements for the UHF solutions of square $\mathrm{H}_{4}$ with a side length of $2.0 \AA$ and $m_{s}=1$. High-spin $(\alpha)$ and low-spin $(\beta)$ orbitals occupy the left and right columns respectively. 
B. $m_{s}=2$

(viii)

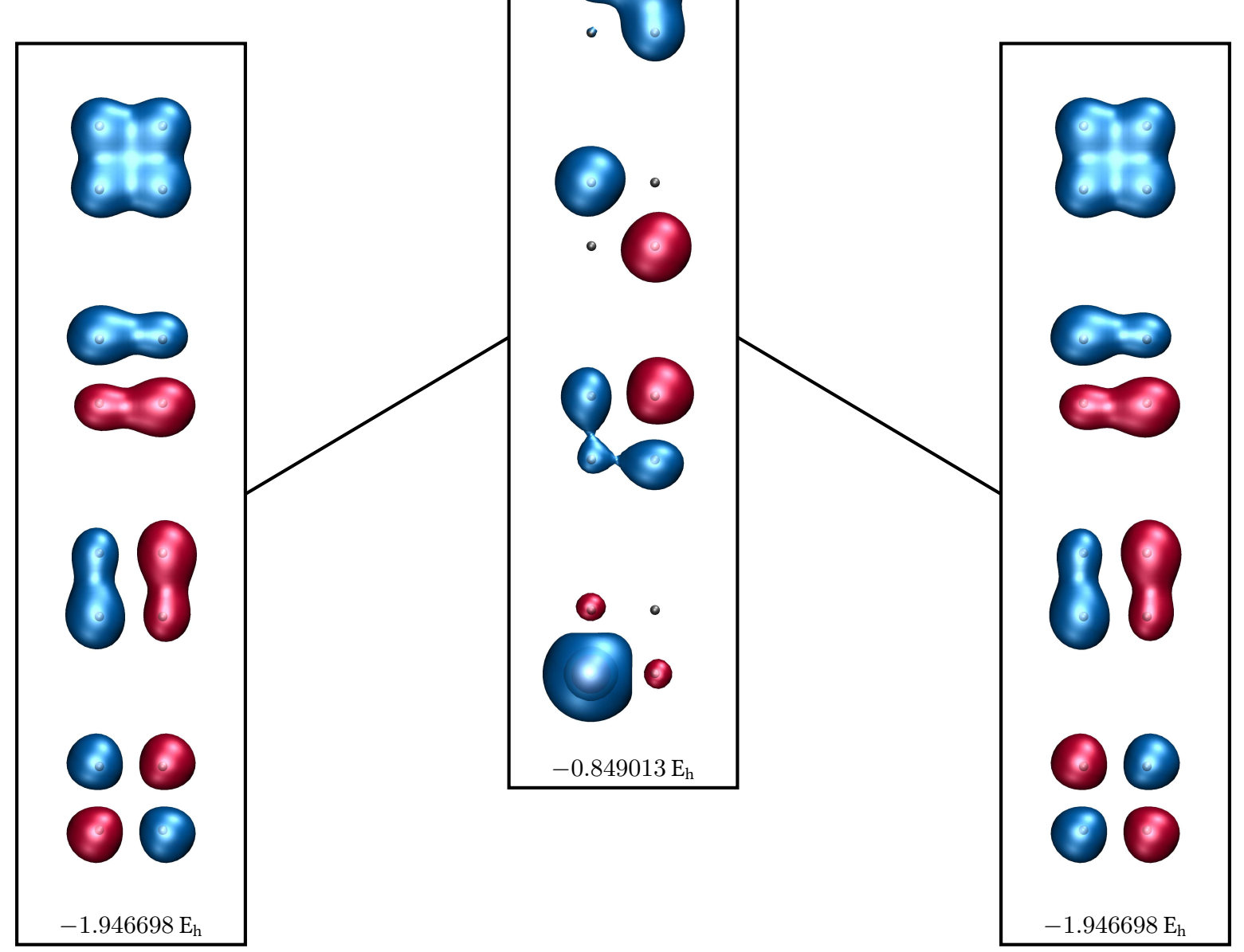

FIG. S2: Illustration of the orbital rearrangement in $\mathrm{H}_{4}$ (3-21G) that inconverts the sign-permuted global UHF minima for $m_{s}=2$. The rearrangement involves a $1 \mathrm{~s} \rightarrow 2$ s transition localised on one hydrogen, giving an unusually high-energy index-1 transition state. All electrons occupy high-spin $(\alpha)$ orbitals, which are arranged in order of increasing energy (top to bottom). 